Mathematical Modelling and Analysis

Volume 22 Number 4, July 2017, 425-440

https://doi.org/10.3846/13926292.2017.1318796

(c) Vilnius Gediminas Technical University, 2017
Publisher: Taylor\&Francis and VGTU

http://www.tandfonline.com/TMMA

ISSN: $1392-6292$

eISSN: 1648-3510

\title{
Special Splines of Hyperbolic Type for the Solutions of Heat and Mass Transfer 3-D Problems in Porous Multi-Layered Axial Symmetry Domain
}

\section{Harijs Kalis ${ }^{1}$, Andris Buikis ${ }^{1}$, Aivars Aboltins ${ }^{2}$ and Ilmars Kangro ${ }^{3}$}

\author{
${ }^{1}$ Institute of Mathematics and Computer Science of University of Latvia \\ Raina bulvāris 29, LV-1459 Rīga, Latvija \\ ${ }^{2}$ Latvia University of Agriculture, Faculty of Engineering \\ 5 J. Cakstes blvd., LV-3001 Jelgava, Latvia \\ ${ }^{3}$ Rezekne Academy of Technologies, Faculty of Engineering \\ Atbrīivoŝanas aleja 115, LV-4601 Rēzekne, Latvija \\ E-mail(corresp.): ilmars.kangro@ru.lv \\ E-mail: kalis@lanet.lv \\ E-mail: buikis@latnet.lv \\ E-mail: aivars.aboltins@llu.lv
}

Received September 23, 2016; revised April 8, 2017; published online July 15, 2017

\begin{abstract}
In this paper we study the problem of the diffusion of one substance through the pores of a porous multi layered material which may absorb and immobilize some of the diffusing substances with the evolution or absorption of heat. As an example we consider circular cross section wood-block with two layers in the radial direction. We consider the transfer of heat process. We derive the system of two partial differential equations (PDEs) - one expressing the rate of change of concentration of water vapour in the air spaces and the other - the rate of change of temperature in every layer. The approximation of corresponding initial boundary value problem of the system of PDEs is based on the conservative averaging method (CAM) with special integral splines. This procedure allows reduce the $3-\mathrm{D}$ axis-symmetrical transfer problem in multi-layered domain described by a system of PDEs to initial value problem for a system of ordinary differential equations (ODEs) of the first order.
\end{abstract}

Keywords: special splines, averaging method, 3D porous axial symmetry domain. 


\section{Introduction}

The task of sufficient accuracy numerical simulation of quick solution 3-D problems for mathematical physics in multi-layered media is important in the known areas of the applied sciences. With regard to the numerical analysis several numerical methods are known for solving 3-D problems: FEM, BEM, spectral methods, multigrids and others methods. For simple engineering calculations conservative averaging method (CAM) with special integral hyperbolic type splines is chosed.

CAM by using integral parabolic type splines was developed by A. Buikis in his Doctoral Thesis [3] (in the Russian language and [5]) and in several papers [4], [1] etc. These methods were applied to create the mathematical simulation of the mass transfer 3-D initial boundary-value problems for parabolic type partial differential equations of second order with piece-wise coefficients in multi-layered underground systems.

In this paper the special spline with two different functions of the hyperbolic type, which interpolate middle integral values of piece-wise smooth function, is used (see [5], [9], [2], [10], [11] ). With the help of these splines the problems of mathematical physics in 3-D with piece-wise coefficients with respect to one coordinate are reduced to problems for system of PDEs in 2-D and 1-D.

If the parameters of the hyperbolic type spline tend to zero, we get the integral parabolic spline, obtained from A. Buikis.

The study of hydrodynamic flow and heat transfer through a porous media becomes much more interesting due to its vast applications for many decades $[15],[17],[20],[19],[6],[7],[8],[18]$. In [19] is considered numerical algorithms for modelling of liquid polymer injection, so that the liquid polymer is flowing through a porous medium. Many mathematical models are developed for the analysis of such processes example mathematical models of moisture movement in wood, when the wood is considered as porous media [13], [14] and [12].

In [13] it is described the moisture movement process in the wood. In the model it is assumed that there is a moisture exchange between two environments and diffusion and convection processes are determining the moisture movement in the wood cells.

In this paper we study the linear heat and moisture transfer processes in the porous multilayered media layer by using CAM with special integral splines. In one layer similar process is analysed and decribed in [15], [16].

\section{The mathematical model}

The process of diffusion is considered in 3-D domain

$$
\Omega=\{(r, z, \phi): 0 \leq r \leq R, \quad 0 \leq z \leq L, \quad 0 \leq \phi \leq 2 \pi\} .
$$

The domain $\Omega$ consist of multilayer medium in the $\mathrm{r}$ direction. We will consider the non-stationary axisymmetrical problem of the linear diffusion theory for multilayered piece-wise homogenous materials of $\mathrm{N}$ layers in the sub domain

$$
\Omega_{i}=\left\{(r, z, \phi): \quad r \in\left(r_{i-1}, r_{i}\right), \quad z \in(0, L), \quad \phi \in(0,2 \pi)\right\}, \quad i=\overline{1, N},
$$


where $H_{i}=r_{i}-r_{i-1}$ is the height of layer $\Omega_{i}, r_{0}=0, r_{N}=R$.

We shall further assume linear dependence on both temperature and moisture content in every layer [15]

$$
M_{i}=\text { const }+\sigma_{i} C_{i}-\omega_{i} T_{i}
$$

where $C_{i}(r, z, t)$ is the concentration of water vapour in the air spaces, $M_{i}$ is the amount of moisture absorbed by unit mass of fibre, $\sigma_{i}$ and $\omega_{i}$ are constants.

We can derive two equations, one expressing the rate of change of concentration and the other - the rate of change of temperature. The water vapour diffusion PDEs in the cylindrical coordinates is in following form

$$
\begin{aligned}
& m_{i}\left(\frac{1}{r}\left(\frac{\partial}{\partial r}\left(D_{i r} r \frac{\partial C_{i}}{\partial r}\right)\right)+D_{i z} \frac{\partial^{2} C_{i}}{\partial z^{2}}\right) \\
& \quad=m_{i} \frac{\partial C_{i}}{\partial t}+\left(1-m_{i}\right) \rho_{s} \frac{\partial M_{i}}{\partial t} ; \quad r \in\left[r_{i-1}, r_{i}\right], \quad i=\overline{1, N}, \quad t>0
\end{aligned}
$$

where $D_{i r}, D_{i z}$ are the diffusion coefficients for moisture in air, $m_{i}$ are the fraction of the total volume of the material occupied by air and $1-m_{i}$ is the fraction of the porous material occupied by fibre of density $\rho_{s}, t$ is the time. If $m_{i}=1$, then the equation is diffusion equation for the concentration without fibres.

The heat diffusion PDEs can be rewritten in the following form:

$$
\begin{aligned}
& c_{i} \rho_{i} \frac{\partial T_{i}}{\partial t}=\frac{1}{r}\left(\frac{\partial}{\partial r}\left(K_{i r} r \frac{\partial T_{i}}{\partial r}\right)\right)+K_{i z} \frac{\partial^{2} T_{i}}{\partial z^{2}} \\
& \quad+q_{i} \rho_{i} \frac{\partial M_{i}}{\partial t} ; \quad r \in\left[r_{i-1}, r_{i}\right], \quad i=\overline{1, N}, \quad t>0
\end{aligned}
$$

where $c_{i}$ are the specific heat of the fibres, $K_{i r}, K_{i z}, \rho_{i}$ are the heat conductivities and the densities of the porous material, $q_{i}$ is the heat evolved when the water vapour is absorbed by the fibres.

We assume that all coefficients in the PDEs are assumed constant and independent of moisture concentration and temperature. By eliminating $M_{i}$ from (2.1), we obtain the system of two PDEs

$$
\begin{aligned}
& D_{i r}^{T} \frac{1}{r}\left(\frac{\partial}{\partial r}\left(r \frac{\partial T_{i}}{\partial r}\right)\right)+D_{i z}^{T} \frac{\partial^{2} T_{i}}{\partial z^{2}}=\frac{\partial T_{i}}{\partial t}-\nu_{i} \frac{\partial C_{i}}{\partial t} \\
& D_{i r}^{C} \frac{1}{r}\left(\frac{\partial}{\partial r}\left(r \frac{\partial C_{i}}{\partial r}\right)\right)+D_{i z}^{C} \frac{\partial^{2} C_{i}}{\partial z^{2}}=\frac{\partial C_{i}}{\partial t}-\lambda_{i} \frac{\partial T_{i}}{\partial t}, \quad i=\overline{1, N}
\end{aligned}
$$

where

$$
\begin{aligned}
& D_{i r}^{T}=\frac{K_{i r}}{\rho_{i}\left(c_{i}+q_{i} \omega_{i}\right)}, \quad D_{i r}^{C}=\frac{D_{i r} m_{i}}{m_{i}+\left(1-m_{i}\right) \rho_{s} \sigma_{i}}, \quad D_{i z}^{T}=\frac{K_{i z}}{\rho_{i}\left(c_{i}+q_{i} \omega_{i}\right)}, \\
& D_{i z}^{C}=\frac{D_{i z} m_{i}}{m_{i}+\left(1-m_{i}\right) \rho_{s} \sigma_{i}}, \quad \nu_{i}=\frac{q_{i} \sigma_{i}}{c_{i}+q_{i} \omega_{i}}, \quad \lambda_{i}=\frac{\left(1-m_{i}\right) \omega_{i} \rho_{s}}{m_{i}+\left(1-m_{i}\right) \rho_{s} \sigma_{i}}, \quad \lambda_{i} \nu_{i}<1 .
\end{aligned}
$$

For the initial condition for $t=0$ we give $T_{i}(r, z, 0)=T_{0}, C_{i}(r, z, 0)=C_{0}, i=$ $\overline{1, N}$, where $T_{0}, C_{0}$ are known constants. 
We use following boundary and continuous conditions:

$$
\begin{aligned}
& \frac{\partial Q_{1}(0, z, t)}{\partial r}=0, \quad Q_{N}(R, z, t)=Q_{a r}, \quad Q_{N}(r, L, t)=Q_{a z}, \frac{\partial Q_{i}(r, 0, t)}{\partial z}=0 \\
& Q_{i}\left(r_{i}, z, t\right)=Q_{i+1}\left(r_{i}, z, t\right), \quad D_{i r}^{Q} \frac{\partial Q_{i}\left(r_{i}, z, t\right)}{\partial r}=D_{i+1, r}^{Q} \frac{\partial Q_{i+1}\left(r_{i}, z, t\right)}{\partial r},
\end{aligned}
$$

where $Q_{i}=Q_{i}(r, z, t), Q=(T ; C)$ are the solutions in every layer, $T_{a r}, T_{a z}$, $C_{a r}, C_{a z}$ are the given temperature and concentration on the boundary.

This problem for the system of PDEs is uniquely solvable and stable for $\lambda_{i} \geq 0, \nu_{i} \geq 0, \lambda_{i} \times \nu_{i}<1$, because then the system (2.2) can be represented in the following way:

$$
\frac{\partial W_{i}}{\partial t}=A_{i r} \frac{1}{r}\left(\frac{\partial}{\partial r}\left(r \frac{\partial W_{i}}{\partial r}\right)\right)+A_{i z} \frac{\partial^{2} W_{i}}{\partial z^{2}},
$$

where $W_{i}=\left(T_{i}, C_{i}\right)$ is 2-order column-vector and

$$
A_{i r}=\frac{1}{1-\lambda_{i} \nu_{i}}\left(\begin{array}{cc}
D_{i r}^{T} & \nu_{i} D_{i r}^{C} \\
\lambda_{i} D_{i r}^{T} & D_{i r}^{C}
\end{array}\right), \quad A_{i z}=\frac{1}{1-\lambda_{i} \nu_{i}}\left(\begin{array}{cc}
D_{i z}^{T} & \nu_{i} D_{i z}^{C} \\
\lambda_{i} D_{i z}^{T} & D_{i z}^{C}
\end{array}\right)
$$

are matrices with positive eigenvalues.

\section{The conservative averaging method in z-direction}

We consider CAM of the special integral splines with hyperbolic trigonometrical functions for solving the 3-D initial boundary-value problem in $z$-direction [10]. This procedure allows reducing the 2 -D problem in $r, z$-directions to a $1 \mathrm{D}$ problem in r-direction. Using CAM in $z$-direction with parameters $a_{i z}$ we have

$$
Q_{i}(r, z, t)=Q_{i v}(r, t)+m_{i}^{Q}(r, t) f_{i z 1}\left(z-\overline{z_{i}}\right)+e_{i}^{Q}(r, t) f_{i z 2}\left(z-\overline{z_{i}}\right),
$$

where $Q_{i v}(r, t)=\frac{1}{L} \int_{0}^{L} Q_{i}(r, z, t) d z, Q_{i}=\left(T_{i}, C_{i}\right), f_{i z 1}(z)=\frac{0.5 L \sinh \left(a_{i z}(z-L / 2)\right)}{\sinh \left(0.5 a_{i z} L\right)}$, $f_{i z 2}(z)=\frac{\cosh \left(a_{i z}(z-L / 2)\right)-A_{i 0}}{8 \sinh ^{2}\left(a_{i z} L / 4\right)}, A_{i 0}=\frac{\sinh \left(a_{i z} L / 2\right)}{a_{i z} L / 2}$. The parameters $a_{i z}$ can be choosen to minimize the maximal error. As the parameters $a_{i z}>0$ tends to zero then as the limit we obtain the integral parabolic spline [2], because

$$
A_{i 0} \rightarrow 1: Q_{i}(r, z, t)=Q_{i, v}(r, t)+m_{i}^{Q}(z-L / 2)+e_{i}^{Q}\left(\frac{(z-L / 2)^{2}}{L^{2}}-\frac{1}{12}\right)
$$

The unknown functions $m_{i}=m_{i}^{Q}(r, t), e_{i}=e_{i}^{Q}(r, t)$ can be determined from conditions (2.3): 1) for $\left.z=0, m_{i} d_{i}-e_{i} k_{i}=0, m_{i}=e_{i} p_{i}, p_{i}=k_{i} / d_{i}, 2\right)$ for $z=L, Q_{a z}=Q_{i v}+m_{i} L / 2+e_{i} b_{i}, e_{i}=\left(Q_{a z}-Q_{i v}\right) / g_{0 i}$, where

$$
\begin{aligned}
& d_{i}=0.5 L a_{i z} \operatorname{coth}\left(0.5 a_{i z} L\right), \quad k_{i}=0.25 a_{i z} \operatorname{coth}\left(0.25 a_{i z} L\right), \\
& b_{i}=\left(\left(\cosh \left(a_{i z} L / 2\right)-A_{i 0}\right) /\left(8 \sinh ^{2}\left(a_{i z} L / 4\right)\right), \quad g_{0 i}=b_{i}+0.5 L p_{i} .\right.
\end{aligned}
$$


Now the 1D initial-value problem (2.2) is in the following form

$$
\left\{\begin{array}{l}
D_{i r}^{T} \frac{1}{r}\left(\frac{\partial}{\partial r}\left(r \frac{\partial T_{i v}}{\partial r}\right)\right)+D_{i z}^{T} a_{0 i}^{2}\left(T_{a z}-T_{i v}\right)=\frac{\partial T_{i v}}{\partial t}-\nu_{i} \frac{\partial C_{i v}}{\partial t} \\
D_{i r}^{C} \frac{1}{r}\left(\frac{\partial}{\partial r}\left(r \frac{\partial C_{i v}}{\partial r}\right)\right)+D_{i z}^{C} a_{0 i}^{2}\left(C_{a z}-C_{i v}\right)=\frac{\partial C_{i v}}{\partial t}-\lambda_{i} \frac{\partial T_{i v}}{\partial t} \\
\frac{\partial T_{1 v}(0, t)}{\partial r}=0, \frac{\partial C_{1 v}(0, t)}{\partial r}=0, T_{N v}(R, t)=T_{a r}, C_{N v}(R, t)=C_{a r} \\
T_{i v}\left(r_{i}, t\right)=T_{i+1, v}\left(r_{i}, t\right), D_{i r}^{T} \frac{\partial T_{i v}\left(r_{i}, t\right)}{\partial r}=D_{i+1, r}^{T} \frac{\partial T_{i+1, v}\left(r_{i}, t\right)}{\partial r}, i=\overline{1, N-1} \\
C_{i v}\left(r_{i}, t\right)=C_{i+1, v}\left(r_{i}, t\right), D_{i r}^{C} \frac{\partial C_{i v}\left(r_{i}, t\right)}{\partial r}=D_{i+1, r}^{C} \frac{\partial C_{i+1, v}\left(r_{i}, t\right)}{\partial r}, i=\overline{1, N-1} \\
T_{i v}(r, 0)=T_{0}, C_{i v}(r, 0)=C_{0}, i=\overline{1, N}
\end{array}\right.
$$

where $a_{0 i}^{2}=2 k_{i} / L g_{0 i}$.

\section{The aprobation of the hyperbolic approximation in $\mathrm{z}-$ direction}

In this section we consider averaging methods with hyperbolic type splines for solving special 3-D boundary-value problem in one layer. For multi-layered domains the accuracy of spline method remained, because of the continuous conditions are fulfilled exactly.

With the help of these splines is reducing the 3 -D problem in $z$-direction to 1-D problem for ODEs. This procedure allows the solution obtained analytically. The spline solution is compared with the analytical solution. We consider the process of diffusion in 3-D parallelepiped

$$
\Omega_{1}=\left\{(x, y, z): 0 \leq x \leq L_{x}, 0 \leq y \leq L_{y}, 0 \leq z \leq L_{z}\right\}
$$

We will find the distribution of stationary concentrations $c=c(x, y, z)$ in $\Omega_{1}$ by solving the following special $3-\mathrm{D}$ boundary value problem for PDE:

$$
\left\{\begin{array}{l}
\frac{\partial}{\partial x}\left(D_{x} \frac{\partial c}{\partial x}\right)+\frac{\partial}{\partial y}\left(D_{y} \frac{\partial c}{\partial y}\right) \\
\quad+\frac{\partial}{\partial z}\left(D_{z} \frac{\partial c}{\partial z}\right)+f_{0} \cos \frac{\pi x}{2 L_{x}} \cos \frac{\pi y}{2 L_{y}}=0 \\
\frac{\partial c(0, y, z)}{\partial x}=\frac{\partial c(x, 0, z)}{\partial y}=0, c\left(L_{x}, y, z\right)=0, c\left(x, L_{y}, z\right)=0 \\
D_{z} \frac{\partial c(x, y, 0)}{\partial z}-\beta_{z}\left(c(x, y, 0)-c_{o} \cos \frac{\pi x}{2 L_{x}} \cos \frac{\pi y}{2 L_{y}}\right)=0 \\
D_{z} \frac{\partial c\left(x, y, L_{z}\right)}{\partial z}+\alpha_{z}\left(c\left(x, y, L_{z}\right)-c_{a} \cos \frac{\pi x}{2 L_{x}} \cos \frac{\pi y}{2 L_{y}}\right)=0
\end{array}\right.
$$

where $f_{0}, c_{o}, c_{a}$ - are fixed constants, $D_{x}, D_{y}, D_{z}$ are the constant diffusion coefficients, $\alpha_{z}, \beta_{z}$ are the constant mass transfer coefficients with the 3 kind boundary conditions. We can obtained the analytical solution of (4.1) in following form: $c(x, y, z)=g(z) \cos \frac{\pi x}{2 L_{x}} \cos \frac{\pi y}{2 L_{y}}$, where the function $g(z)$ is solution of boundary value problem for following ODEs

$$
\left\{\begin{array}{l}
g^{\prime \prime}(z)-a_{0}^{2} g(z)+f_{1}=0 \\
g^{\prime}(0)-\beta\left(g(0)-c_{o}\right)=0, g^{\prime}\left(L_{z}\right)+\alpha\left(g\left(L_{z}\right)-c_{a}\right)=0
\end{array}\right.
$$


where $f_{1}=f_{0} / D_{z}, \beta=\beta_{z} / D_{z}, \alpha=\alpha_{z} / D_{z}, a_{0}^{2}=\pi^{2}\left(D_{y} / L_{y}^{2}+D_{x} / L_{x}^{2}\right) / 4 D_{z}$. We have the following solutions

$$
g(z)=C_{1} \sinh \left(a_{0} z\right)+C_{2} \cosh \left(a_{0} z\right)+f_{2},
$$

where $C_{1}=\beta / a_{0}\left(C_{2}+f_{2}-c_{o}\right), C_{2}=\frac{\left(c_{a}-f_{2}\right)\left(\alpha / a_{0}+\beta / a_{0} c_{3}\right)}{\beta / a_{0} c_{3}+c_{4}}, f_{2}=f_{1} / a_{0}^{2}, c_{3}=$ $\cosh \left(a_{0} L_{z}\right)+\alpha / a_{0} \sinh \left(a_{0} L_{z}\right), c_{4}=\sinh \left(a_{0} L_{z}\right)+\alpha / a_{0} \cosh \left(a_{0} L_{z}\right)$.

Next we use the averaged method for (4.2) with fixed parametrical functions $f_{z 1}, f_{z 2} \cdot g(z)=g_{a}+m f_{z 1}\left(z-L_{z} / 2\right)+e f_{z 2}\left(z-L_{z} / 2\right)$, where $g_{a}=\frac{1}{L_{z}} \int_{0}^{L_{z}} g(z) d z$ is the averaged value, $\int_{0}^{L_{z}} f_{z 1} d z=\int_{0}^{L_{z}} f_{z 2} d z=0, f_{z 1}=\frac{0.5 L_{z} \sinh \left(a_{z}\left(z-L_{z} / 2\right)\right)}{\sinh \left(a_{z} L_{z} / 2\right)}$, $f_{z 2}=\frac{\cosh \left(a_{z}\left(z-L_{z} / 2\right)\right)-A_{0}}{8 \sinh ^{2}\left(a_{z} L_{z} / 4\right)}, A_{0}=\sinh \left(a_{z} L_{z} / 2\right) /\left(a_{z} L_{z} / 2\right), a_{z}=a_{0}$ is the optimal parameter.

If the parameters $a_{z}$ tend to zero then the limit is the integral parabolic spline [4]. The unknown coefficients $m, e$ we can determine from boundary conditions (4.2):

1) for $\mathrm{z}=0, m d-e k-\beta\left(g_{a}-0.5 m L_{z}+e b-c_{o}\right)=0$,

2) for $\mathrm{z}=L_{z}, m d+e k+\alpha\left(g_{a}+0.5 m L_{z}+e b-c_{a}\right)=0$, where $d=\left(a_{z} L_{z} / 2\right) \operatorname{coth}\left(a_{z} L_{z} / 2\right), k=\left(a_{z} / 4\right) \operatorname{coth}\left(a_{z} L_{z} / 4\right), b=\frac{\left.\cosh \left(a_{z} L_{z} / 2\right)\right)-A_{0}}{8 \sinh ^{2}\left(a_{z} L_{z} / 4\right)}$.

Now the-boundary value problem (4.2) can be written in the form

$$
1 / L_{z}\left(g^{\prime}\left(L_{z}\right)-g^{\prime}(0)\right)-a_{0}^{2} g_{a}+f_{1}=0, g^{\prime}\left(L_{z}\right)-g^{\prime}(0)=2 e k
$$

or

$$
g_{a}=\left(f_{1} L_{z}+2 d a_{e}\right) /\left(a_{0}^{2} L_{z}+2 k g_{e}\right),
$$

where the $a_{e}$ and $g_{e}$ are given constants.

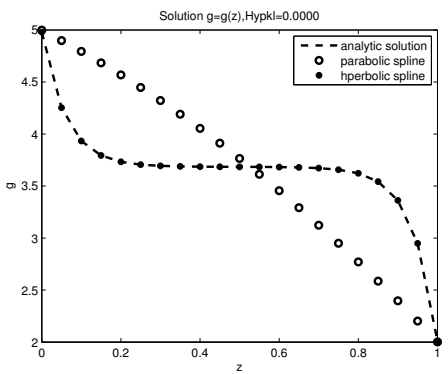

(a) $g(z)$

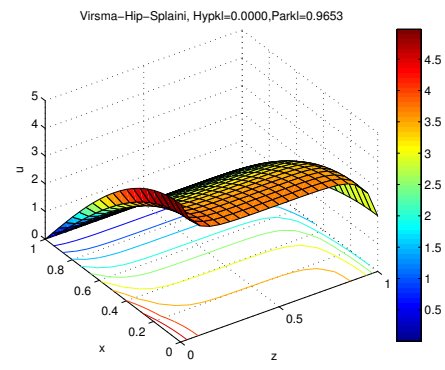

(b) $c(x, 0, z)$

Figure 1. Solutions

Example 1. We consider the following parameters: $L_{x}=L_{y}=L_{z}=1, f_{0}=0.1$, $\alpha_{z}=0.3, \beta_{z}=0.1, c_{o}=5, c_{a}=2, D_{x}=10^{-2}, D_{y}=10^{-3}, D_{z}=10^{-4}$, $a_{0}=16.4747$.

We have following maximal errors: for hyperbolic spline $e r_{h}=2.10^{-9}$, for parabolic $e r_{p}=0.9653$ (see $g(z)$ in Figure 1a and $c(x, 0, z)$ in Figure 1b). If $D_{y}=0, y=0$, then $e r_{h}=8.10^{-8}, e r_{p}=1.0423$. 


\section{The aprobation of the hyperbolic approximation in $\mathrm{r}$ - direction}

The process of diffusion is considered in 3-D cylinder

$$
\Omega_{2}=\left\{(r, z, \phi): 0 \leq r \leq R, 0 \leq z \leq L_{z}, 0 \leq \phi \leq 2 \pi\right\} .
$$

We solve the stationary boundary value problem with axial symmetry and find the distribution of concentrations $c=c(r, z)$ in $\Omega_{2}$ by solving the following special boundary value problem for PDE:

$$
\begin{aligned}
& D_{r} \frac{1}{r} \frac{\partial}{\partial r}\left(r \frac{\partial c}{\partial r}\right)+\frac{\partial}{\partial z}\left(D_{z} \frac{\partial c}{\partial z}\right)+f_{0} \cos \frac{\pi z}{2 L_{z}}=0, \\
& \frac{\partial c(r, 0)}{\partial z}=\frac{\partial c(0, z)}{\partial r}=0, \quad c\left(r, L_{z}\right)=0 \\
& D_{r} \frac{\partial c(R, z)}{\partial r}+\alpha_{r}\left(c(R, z)-c_{a} \cos \frac{\pi z}{2 L_{z}}\right)=0,
\end{aligned}
$$

where $f_{0}, c_{a}$ - are the fixed constants, $D_{r}, D_{z}$ are the diffusion coefficients, $\alpha_{r}$ is the constant mass transfer coefficient. We can obtaine the analytical solution of (5.1) in following form: $c(r, z)=g(r) \cos \left(\pi z / 2 L_{z}\right)$, where the function $g(r)$ is solution of boundary value problem for ODE

$$
\begin{aligned}
& g^{\prime \prime}(r)+\frac{1}{r} g^{\prime}(r)-a_{0}^{2} g(r)+f_{1}=0, \\
& g^{\prime}(0)=0, \quad g^{\prime}(R)+\alpha\left(g(R)-c_{a}\right)=0,
\end{aligned}
$$

where $f_{1}=f_{0} / D_{r}, \alpha=\alpha_{r} / D_{r}, a_{0}^{2}=\frac{\pi^{2} D_{z} / L_{z}^{2}}{4 D_{r}}$. We have following solutions

$$
g(r)=C_{1} I_{0}\left(a_{0} r\right)+f_{2},
$$

where $f_{2}=\frac{f_{1}}{a_{0}^{2}}, C_{1}=\frac{\alpha\left(c_{a}-f_{2}\right)}{a_{0} I_{1}\left(a_{0} R\right)+\alpha I_{0}\left(a_{0} R\right)}, I_{0}, I_{1}$ are the modified Bessel functions. Next we use the averaged method for (5.2) with fixed parametrical functions $f_{r 1}, f_{r 2}, g_{1}(r)=g_{a}+m f_{r 1}(r-R / 2)+e f_{r 2}(r-R / 2)$, where $g_{a}=\frac{2}{R^{2}} \int_{0}^{R} r g(r) d r$ is the averaged value, $\int_{0}^{R} r f_{r 1} d r=\int_{0}^{R} r f_{r 2} d r=0, f_{r 1}=\frac{R^{2} a_{r}^{2} \sinh \left(a_{r}(r-R / 2)\right)}{4 \sinh \left(a_{r} R / 2\right)(d-1)}-1$, $f_{r 2}=\frac{\cosh \left(a_{r}(r-R / 2)\right)-A_{0}}{8 \sinh ^{2}\left(a_{r} R / 4\right)}, A_{0}=\sinh \left(a_{r} R / 2\right) /\left(a_{r} R / 2\right), d=R a_{r} / 2 \operatorname{coth}\left(a_{r} R / 2\right)$, $a_{r}=a_{0}+k o r, k o r$ is the correction for optimal parameter. The unknown correction kor can be obtained with Matlab using the operators "fminbnd, fplot" by minimizing the integrals $I_{2}(k o r)=\int_{0}^{R}\left(g(r)-g_{1}(r)\right)^{2} d r$ or $I_{1}(k o r)=$ $\int_{0}^{R}\left|g(r)-g_{1}(r)\right| d r$.

We can see that the parameters $a_{r}$ tend to zero then the limit is the integral parabolic spline. The unknown coefficients $m, e$ can be determined from boundary conditions (3.1):

1) for $r=0, m d_{r}-e k=0$,

2) for $r=R, m d_{r}+e k+\alpha\left(g_{a}+m b_{m}+e b_{e}-c_{a}\right)$,

where $k=\left(a_{r} / 4\right) \operatorname{coth}\left(a_{r} R / 4\right), d_{r}=0.5 R d a_{r}^{2} /(d-1), b_{e}=\frac{\left.\cosh \left(a_{r} R / 2\right)\right)-A_{0}}{8 \sinh ^{2}\left(a_{r} R / 4\right)}$, $b_{m}=\frac{R^{2} a_{r}^{2}}{4(d-1)}-1$. 
Therefore $e=\left(c_{a}-g_{a}\right) / g_{1}, m=e k / d_{r}, g_{1}=2 k / \alpha+b_{m} k / d_{r}+b_{e}$. Now the-boundary value problem (5.2) is given in the form

$$
2 / R^{2}\left(R g^{\prime}(R)\right)-a_{0}^{2} g_{a}+f_{1}=0, g^{\prime}(R)=2 e k
$$

or

$$
g_{a}=\left(f_{1} g_{1} R+4 k c_{a}\right) /\left(4 k+a_{0}^{2} g_{1} R\right) .
$$

Example 2. We consider the following parameters $L_{z}=R=1, f_{0}=$ $-0.1, \alpha_{r}=10.01, c_{a}=10, D_{r}=10^{-2}, D_{z}=10^{-2}, a_{0}=1.5708$. We get following maximal errors: for hyperbolic spline $\mathrm{er}_{h}=0.00084$, $\mathrm{kor}=-0.200$ for parabolic $e r_{p}=0.7476$ (see $g(r)$ in Figure 2a and $c(r, z)$ in Figure $2 \mathrm{~b}$ ).

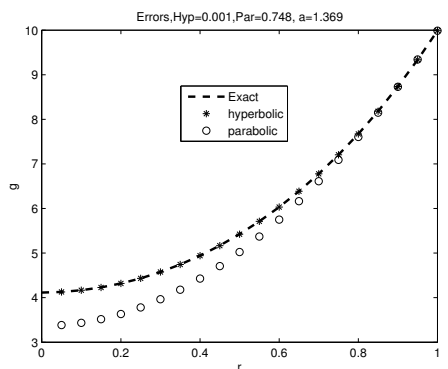

(a) $g(r)$

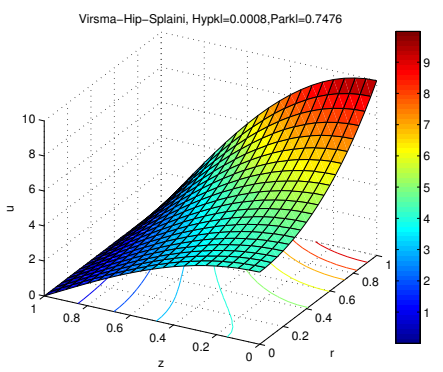

(b) $c(r, z)$

Figure 2. Solutions

We have piecewise linear graphic for integral $I_{1}$ in the interval $[-0.4,0]$ and parabolic type for $I_{2}$. The minimal values for integrals are $I_{1}=6.12 e-4$, $I_{2}=6.9 e-7$. If $\alpha_{r}=0.01$ then $e r_{h}=0.00032, e r_{p}=0.3412$, then the minimal values for integrals are $I_{1}=3.14 e-4, I_{2}=1.7 e-7$, but for $f_{0}=1.01$ we have $e r_{h}=0.00079, e r_{p}=0.8412$ and the minimal values for integrals are $I_{1}=6.9 e-4, I_{2}=8.25 e-7$.

\section{$6 \quad \mathrm{CAM}$ in $r$-direction for $N$-layers}

Using averaged method with parameters $a_{i r}^{Q}$ we have

$$
Q_{i v}(r, t)=Q_{i v v}(t)+m_{i r}^{Q}(t) f_{i r 1}(r)+e_{i z}^{Q}(t) f_{i r 2}(r),
$$

where $Q_{i v v}(t)=\frac{1}{H_{i} \overline{r_{i}}} \int_{r_{i-1}}^{r_{i}} r Q_{i v}(r, t) d r, f_{i r 1}(r)=\frac{0.5 H_{i} \overline{r_{i}}\left(a_{i r}^{Q}\right)^{2} \sinh \left(a_{i r}^{Q}\left(r-\overline{r_{i}}\right)\right)}{\sinh \left(0.5 a_{i r}^{Q} H_{i}\right)\left(d_{i}-1\right)}-1$, $f_{i r 2}(r)=\frac{\cosh \left(a_{i r}^{Q}\left(r-\overline{r_{i}}\right)-A_{i 1}^{Q}\right.}{8 \sinh ^{2}\left(a_{i r}^{Q} H_{i} / 4\right)}, \overline{r_{i}}=\left(r_{i-1}+r_{i}\right) / 2, r \in\left[r_{i-1}, r_{i}\right], A_{i 1}^{Q}=\frac{\sinh \left(a_{i r}^{Q} H_{i} / 2\right)}{a_{i r}^{Q} H_{i} / 2}$, $d_{i}^{Q}=0.5 H_{i} a_{i r}^{Q} \operatorname{coth}\left(0.5 a_{i r}^{Q} H_{i}\right), i=\overline{1, N}$. We have used the following values of parameters $a_{i r}^{T}=a_{0 i} \sqrt{D_{i z}^{T} / D_{i r}^{T}}, a_{i r}^{C}=a_{0 i} \sqrt{D_{i z}^{C} / D_{i r}^{C}}$.

We can see that if parameters $a_{i r}^{Q}>0$ tend to zero, then the limit is the integral parabolic spline:

$$
Q_{i v}(r, t)=Q_{i v v}+m_{i r}^{Q}\left(\frac{12 \overline{r_{i}}}{H_{i}^{2}}\left(r-\overline{r_{i}}\right)-1\right)+e_{i r}^{Q}\left(\frac{\left(r-\overline{r_{i}}\right)^{2}}{H_{i}^{2}}-\frac{1}{12}\right) .
$$


From boundary conditions (2.3) we get the linear system of $2 N$-equations for determination unknown functions $m_{i}=m_{i r}^{Q}(t), e_{i}=e_{i r}^{Q}(t)$ :

1) for $r=0, m_{1} d_{1 r}^{Q}-e_{1} k_{1 r}^{Q}=0$,

2) for $r=R, Q_{a r}=Q_{N v v}+m_{N} b_{N m}+e_{N} b_{N e}$,

3) for $r=r_{i}, Q_{i v v}+m_{i} b_{i m}+e_{i} b_{i e}=Q_{i+1, v v}-m_{i+1}\left(b_{i+1, m}+2\right)+e_{i+1} b_{i+1, e}$, $D_{i r}^{Q}\left(m_{i} d_{i r}^{Q}+e_{i} k_{i r}^{Q}\right)=D_{i+1, r}^{Q}\left(m_{i+1} d_{i+1, r}^{Q}-e_{i+1} k_{i+1, r}^{Q}\right), i=\overline{1, N-1}$, where

$$
\begin{aligned}
& d_{i r}^{Q}=d_{i}^{Q} \overline{r_{i}}\left(a_{i r}^{Q}\right)^{2} /\left(d_{i}^{Q}-1\right), \quad k_{i r}^{Q}=0.25 a_{i r}^{Q} \operatorname{coth}\left(0.25 a_{i r}^{Q} H_{i}\right), \\
& b_{i e}^{Q}=\left(\left(\cosh \left(a_{i r}^{Q} H_{i} / 2\right)-A_{i 1}^{Q}\right) /\left(8 \sinh ^{2}\left(a_{i r}^{Q} H_{i} / 4\right)\right), b_{i m}^{Q}=\frac{0.5 H_{i} \overline{r_{i}}\left(a_{i r}^{Q}\right)^{2}}{d_{i}^{Q}-1}-1 .\right.
\end{aligned}
$$

Now the 1D initial- value problem (3.1) gives the following ODEs system:

$$
\left\{\begin{array}{l}
D_{i r}^{T}\left(m_{i r}^{T}(t) d_{i r}^{T} / \overline{r_{i}}+2 e_{i r}^{T}(t) k_{i r}^{T} / H_{i}\right)+D_{i z}^{T}\left(a 0_{i}^{T}\right)^{2}\left(T_{a z}-T_{i v v}(t)\right) \\
=\frac{\partial T_{i v v}(t)}{\partial t}-\nu_{i} \frac{\partial C_{i v v}(t)}{\partial t} \\
D_{i r}^{C}\left(m_{i r}^{C}(t) d_{i r}^{C} / \bar{r}_{i}+2 e_{i r}^{C}(t) k_{i r}^{C} / H_{i}\right)+D_{i z}^{C}\left(a 0_{i}^{C}\right)^{2}\left(C_{a z}-C_{i v v}(t)\right) \\
=\frac{\partial C_{i v v}(t)}{\partial t}-\lambda_{i} \frac{\partial T_{i v v}(t)}{\partial t} \\
T_{i v v}(0)=T_{0}, C_{i v v}(0)=C_{0}, \quad i=\overline{1, N}
\end{array}\right.
$$

\section{CAM for two layers}

For $N=2$ we get from (6.1) the following ODE initial-value problem:

$$
\left\{\begin{array}{l}
b_{i 1}^{T} T_{1 v v}(t)+b_{i 2}^{T} T_{2 v v}(t)+T_{i 0}=\frac{\partial T_{i v v}(t)}{\partial t}-\nu_{i} \frac{\partial C_{i v v}(t)}{\partial t} \\
b_{i 1}^{C} C_{1 v v}(t)+b_{i 2}^{C} C_{2 v v}(t)+C_{i 0}=\frac{\partial C_{i v v}(t)}{\partial t}-\lambda_{i} \frac{\partial T_{i v v}(t)}{\partial t} \\
T_{i v v}(0)=T_{0}, \quad C_{i v v}(0)=C_{0}, \quad i=1,2
\end{array}\right.
$$

or

$$
\left\{\begin{array}{c}
\dot{T}_{i v v}(t)=\frac{1}{\left(1-\lambda_{i} \nu_{i}\right)}\left(b_{i 1}^{T} T_{1 v v}(t)+b_{i 2}^{T} T_{2 v v}(t)+T_{i 0}+\nu_{i}\left(b_{i 1}^{C} C_{1 v v}(t)\right.\right. \\
\left.\left.\quad+b_{i 2}^{C} C_{2 v v}(t)+C_{i 0}\right)\right), \\
\dot{C}_{i v v}(t)=\frac{1}{\left(1-\lambda_{i} \nu_{i}\right)}\left(\lambda_{i}\left(b_{i 1}^{T} T_{1 v v}(t)+b_{i 2}^{T} T_{2 v v}(t)+T_{i 0}\right)+b_{i 1}^{C} C_{1 v v}(t)\right. \\
\left.+b_{i 2}^{C} C_{2 v v}(t)+C_{i 0}\right), \quad T_{i v v}(0)=T_{0}, \quad C_{i v v}(0)=C_{0},
\end{array}\right.
$$

where $b_{i k}^{Q}, k=1,2, Q_{i 0}, i=1,2, Q=(T, C)$ are known coefficients. We rewrite the system of ODEs (7.2) in the following vector form:

$$
\dot{W}(t)-A W(t)=F, \quad W(0)=W_{0},
$$

where $W(t), W_{0}, F$ are 4 th order vector-columns with elements $\left(T_{1 v v}(t), T_{2 v v}(t)\right.$, $\left.C_{1 v v}(t), C_{2 v v}(t)\right),\left(T_{0}, T_{0}, C_{0}, C_{0}\right)$ and $F_{1}=G_{1}\left(T_{10}+\nu_{1} C_{10}\right), F_{2}=G_{2}\left(T_{20}+\right.$ $\left.\nu_{2} C_{20}\right), F_{3}=G_{1}\left(\lambda_{1} T_{10}+C_{10}\right), F_{4}=G_{2}\left(\lambda_{2} T_{20}+C_{20}\right), G_{1}=\frac{1}{\left(1-\lambda_{1} \nu_{1}\right)}, G_{2}=$ $\frac{1}{\left(1-\lambda_{2} \nu_{2}\right)}$. $A$ is the 4-order matrix

$$
A=\left(\begin{array}{cccc}
G_{1} b_{11}^{T} & G_{1} b_{12}^{T} & G_{1} \nu_{1} b_{11}^{C} & G_{1} \nu_{1} b_{12}^{C} \\
G_{2} b_{21}^{T} & G_{2} b_{22}^{T} & G_{2} \nu_{2} b_{21}^{C} & G_{2} \nu_{2} b_{22}^{C} \\
G_{1} \lambda_{1} b_{11}^{T} & G_{1} \lambda_{1} b_{12}^{T} & G_{1} b_{11}^{C} & G_{1} b_{12}^{C} \\
G_{2} \lambda_{2} b_{21}^{T} & G_{2} \lambda_{2} b_{22}^{T} & G_{2} b_{21}^{C} & G_{2} b_{22}^{C}
\end{array}\right)
$$


The averaged solution is $W(t)=\exp (A t) W_{0}-(E-\exp (A t)) A^{-1} F, Q_{i v}(r, t)=$ $Q_{i v v}(t)+m_{i r}^{Q}(t) f_{i r 1}(r)+e_{i z}^{Q}(t) f_{i r 2}(r)$, where $i=1,2, Q=(T, C)$.

\section{The conservative averaging method for model equations}

In order to apply the averaging method in 2 layers in $r$-direction and estimate the parameter $a_{i r}=a_{i r}^{T}$ we consider the stationary 1-D boundary-value problem (3.1) only for temperature $T=T(r)$ with $\nu=0, T_{a z}=0, T_{a r}=T_{0}, D_{i z}^{T}=1$, $D_{i r}^{T}=D_{i}, i=1,2$ :

$$
\left\{\begin{array}{l}
D_{1} \frac{1}{r}\left(r T_{1}^{\prime}(r)\right)^{\prime}-a_{01}^{2} T_{1}(r)=F_{1}, r \in\left[0, H_{1}\right], \quad T_{1}^{\prime}(0)=0, \\
D_{2} \frac{1}{r}\left(r T_{2}^{\prime}(r)\right)^{\prime}-a_{02}^{2} T_{2}(r)=F_{2}, \quad T_{2}(R)=T_{0}, r \in\left[H_{1}, R\right], \\
T_{1}\left(H_{1}\right)=T_{2}\left(H_{1}\right), \quad D_{1} T_{1}^{\prime}\left(H_{1}\right)=D_{2} T_{2}^{\prime}\left(H_{1}\right), \quad 0<H_{1}<R,
\end{array}\right.
$$

where $T_{0}, F_{i}, a_{0 i}>0, D_{i}>0, i=1,2$ are given constants. The analytical solution is defined as $T_{1}(r)=C_{1} I_{0}\left(a_{1} r\right)-f_{1}, T_{2}(r)=C_{2} I_{0}\left(a_{2} r\right)+C_{3} K_{0}\left(a_{2} r\right)-$ $f_{2}$, where $f_{i}=F_{i} / a_{0 i}^{2}, a_{i}=a_{0 i} / \sqrt{D_{i}}, i=1,2$. The unknown constants are obtained from BCs $\left(I_{0}(r)^{\prime}=I_{1}(r), K_{0}(r)^{\prime}=-K_{1}(r)\right)$ :

$$
\begin{aligned}
& C_{2}=\frac{\left(T_{0}+f_{2}\right)-C_{3} K_{0}\left(a_{2} R\right)}{I_{0}\left(a_{2} R\right)}, \quad C_{3}=\frac{b_{1}-f_{a}}{b_{3}}, \quad b_{1}=\frac{b_{0}\left(T_{0}+f_{2}\right)}{I_{0}\left(a_{2} R\right)}, \\
& b_{0}=D_{2,1} a_{2} I_{0}\left(a_{1} H_{1}\right) I_{1}\left(a_{2} H_{1}\right)-a_{1} I_{1}\left(a_{1} H_{1}\right) I_{0}\left(a_{2} H_{1}\right), f_{a}=a_{1} I_{1}\left(a_{1} H_{1}\right)\left(f_{1}-f_{2}\right), \\
& C_{1}=D_{2,1}\left(C_{2} a_{2} I_{1}\left(a_{2} H_{1}\right)-C_{3} a_{2} K_{1}\left(a_{2} H_{1}\right)\right) /\left(a_{1} I_{1}\left(a_{2} H_{1}\right)\right), D_{2,1}=D_{2} / D_{1}, \\
& b_{3}=b_{0} K_{0}\left(a_{2} R\right) / I_{0}\left(a_{2} R\right)+b_{2}, \quad b_{2}=D_{2,1} a_{2} I_{0}\left(a_{1} H_{1}\right) K_{1}\left(a_{2} H_{1}\right) \\
& \quad+a_{1} I_{1}\left(a_{1} H_{1}\right) K_{0}\left(a_{2} H_{1}\right) .
\end{aligned}
$$

Here $I_{0}, K_{0}, I_{1}, K_{1}$ are the modified Bessel functions.

With hyperbolic averaging method in two layers we have:

$$
S_{i}(r)=T_{i v}+m_{i}\left(\frac{0.5 H_{i} \bar{r}_{i} a_{i r}^{2} \sinh \left(a_{i r}\left(r-\overline{r_{i}}\right)\right)}{\sinh \left(0.5 a_{i r} H_{i}\right)\left(d_{i}-1\right)}-1\right)+e_{i}\left(\frac{\cosh \left(a_{i r}\left(r-\overline{r_{i}}\right)-A_{i}\right.}{8 \sinh ^{2}\left(a_{i r} H_{i} / 4\right)}\right),
$$

where $i=1,2, \overline{r_{1}}=0.5 H_{1}, \overline{r_{2}}=0.5\left(H_{1}+R\right), A_{i}=\frac{\sinh \left(a_{i r} H_{i} / 2\right)}{a_{i r} H_{i} / 2}, d_{i}=$ $0.5 H_{i} a_{i r} \operatorname{coth}\left(0.5 a_{i r} H_{i}\right)$. The unknown parameters are $a_{i r}=a_{i}+k o r_{i}$, where $k \mathrm{kr}_{i}, i=1,2$ are the corrections for the minimal error.

The unknown constants $T_{i v}$ can be determined from stationary equations (7.1) for $i=1,2$ by solving the system of linear equations with respect to $T_{1 v}, T_{2 v}$. The unknown corrections can be obtained with Matlab using the operators "fminsearch, contour" by minimizing the integrals

$$
\begin{aligned}
& I_{2}\left(\text { kor }_{1}, \text { kor }_{2}\right)=\int_{0}^{H_{1}}\left(T_{1}(r)-S_{1}(r)\right)^{2} d r+\int_{H_{1}}^{R}\left(T_{2}(r)-S_{2}(r)\right)^{2} d r \\
& I_{1}\left(k_{0} r_{1}, k_{0}\right)=\int_{0}^{H_{1}}\left|T_{1}(r)-S_{1}(r)\right| d r+\int_{H_{1}}^{R}\left|T_{2}(r)-S_{2}(r)\right| d r
\end{aligned}
$$


Using Matlab for $D_{1}=10^{-2}, D_{2}=10^{-1}, F_{1}=-2, F_{2}=0, a_{01}=2, a_{02}=6$, $a_{1}=20, a_{2}=18.9737, T_{0}=4, R=3, H_{1}=1.8$ we have the following maximal errors:

1) 2.6275 for the parabolic spline $\left(a_{1}=a_{2}=10^{-4}\right)$,

2) 0.0631 for the hyperbolic spline with optimal corrections $k o r_{1}=-0.2838$, kor $_{2}=-0.1700$.

The minimal value for integral $I_{1}$ is 0.0255 , see Figs. $3 \mathrm{a}$ and $3 \mathrm{~b}$.

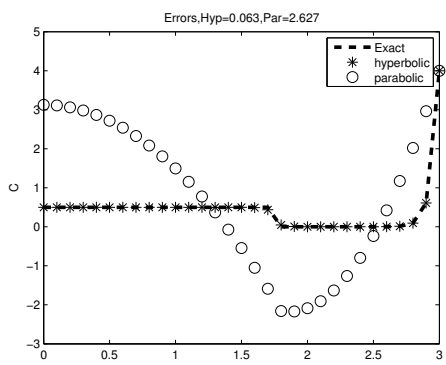

(a) Solution of model equations in 2 layers

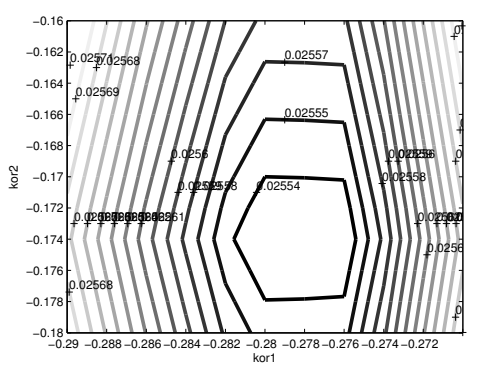

(b) Levels for minimizing in 2 layers

Figure 3. Solution of model equations and levels for minimizing in 2 layers

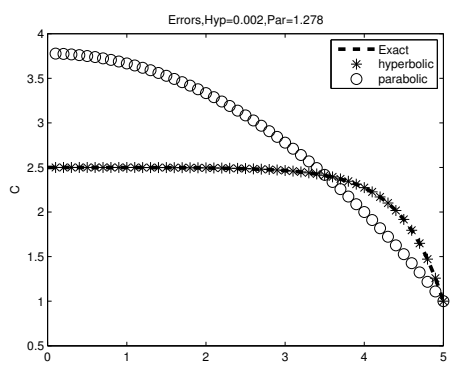

(a) Solution of model equations in 1 layer

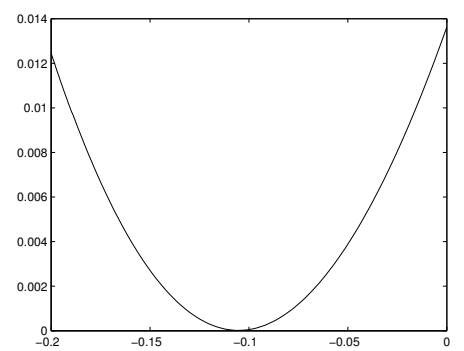

(b) Minimizing function depending on kor in 1 layer

Figure 4. Solution of model equations and minimizing function depending on kor in 1 layer

For one layer we have:

$$
T_{1}(r)=C_{1} I_{0}\left(a_{1} r\right)-f_{1}, C_{1}=\left(T_{0}+f_{1}\right) / I_{0}\left(a_{1} R\right), H_{1}=R .
$$

The unknown correction kor can be obtained with Matlab using the operators "fminbnd, fplot" by minimizing the integrals $I_{2}(k o r)=\int_{0}^{R}\left(T_{1}(r)-S_{1}(r)\right)^{2} d r$ or $I_{1}(k o r)=\int_{0}^{R}\left|T_{1}(r)-S_{1}(r)\right| d r$. For $D_{1}=1, F_{1}=-10, a_{01}=2, a_{1}=1.89$, $T_{0}=1, R=5$ we have the following maximal errors:

1) 1.278 for parabolic spline $\left(a_{1}=10^{-4}\right)$, 
2) 0.0021 for hyperbolic spline with optimal correction $k o r=-0.11$, see Figure 4a and minimizing function with minimal value for integral $I_{2}$ is $5.10^{-5}$ see Figure $4 b$.

In Figs. 5a, 5b we present the spline functions $f_{1}(r)=f_{i r 1}(r), f_{2}(r)=$ $f_{i r 2}(r)$ for $i=1, \overline{r_{i}}=R / 2, R=1$ with $a=a_{i r} \in[0,25]$.

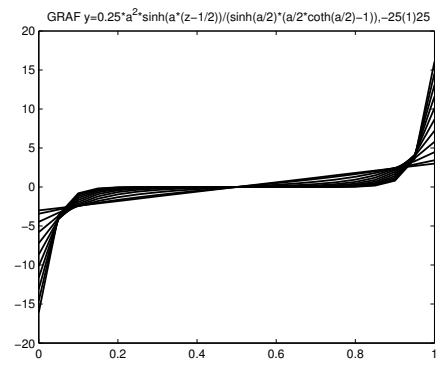

(a) $f_{1}(r)$

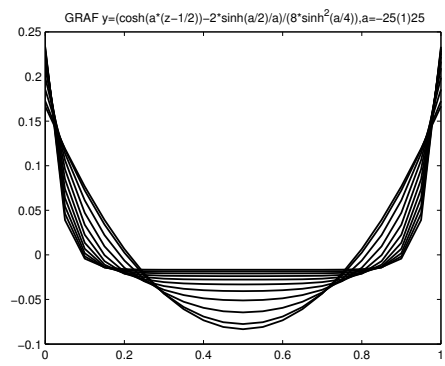

(b) $f_{2}(r)$

Figure 5. Functions for cylindr. coord. depending on $a \in[0,25], R=1$

\section{Some numerical results}

The results of calculations are obtained by MATLAB. We use the discrete values $r_{j}=j h_{r}, j=\overline{0, N_{r}}, N_{r} h_{r}=R, z_{k}=k h_{z}, k=\overline{0, N_{z}}, N_{z} h_{z}=L, t_{n}=n \tau$, $n=\overline{0, N_{t}}, N_{t} \tau=t_{f}, N_{t}=1000, N_{r}=30, N_{z}=10, t_{f}=1000-20000 \mathrm{~s}$.

We consider the drying process in the two laered wood-block (see Figure $6 \mathrm{~b}$ ).

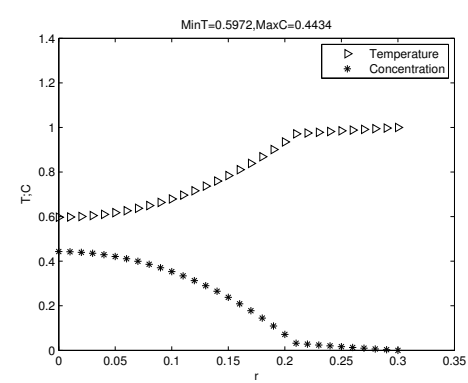

(a) the $T_{v}, C_{v}$ for drying process depending on $r$ by $t=1000$

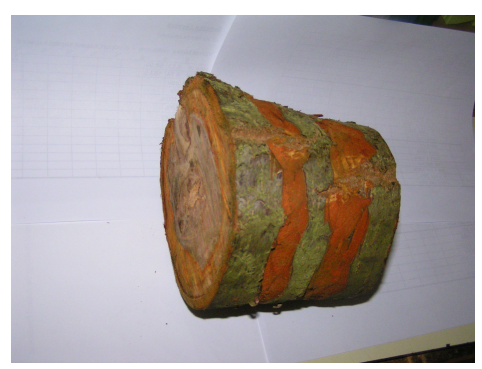

(b) the wood-block

Figure 6. The $T_{v}, C_{v}$ for drying process depending on $r$ by $t=1000$ and the wood-block

The following parameters and diffusion coefficients are used in computations: $L=0.5 m, R=0.3 m, H_{1}=0.21 m, H_{2}=0.09 m, D_{1 z}^{C}=10^{-5}, D_{2 z}^{C}=$ $10^{-4}, D_{1 r}^{C}=10^{-7}, D_{2 r}^{C}=10^{-6}, D_{2 z}^{T}=D_{1 z}^{T}=D_{2 r}^{T}=D_{1 r}^{T}=10^{-3}\left(\frac{m^{2}}{s}\right)$.

The dimensionless value for temperature is defined as $T=\frac{T^{\prime}-T_{0}^{\prime}}{T_{\max }^{\prime}-T_{0}^{\prime}}$ and for the concentration of water vapour $C=\frac{C^{\prime}}{C_{\max }^{\prime}}$, where $T_{0}^{\prime}=20^{0} C, T_{\max }^{\prime}=80^{\circ} \mathrm{C}$, 
$C_{\max }^{\prime}=20 \%, T^{\prime}\left[{ }^{0} C\right], C^{\prime}[\%]$ are the corresponding dimensional values.

For the drying process we have used the following initial and boundary conditions:

$$
T_{0}=0, \quad C_{0}=1, \quad C_{0 r}=C_{0 z}=0, \quad T_{0 r}=T_{0 z}=1 .
$$

The results of calculation for $\lambda=0, \nu=1, a_{1 z}=10, a_{2 z}=1$ are represented in the Table 1 and Figs. (7a)-(8a), where $M C_{v}, M C$ are the maximal values of concentration $C_{v}, C$ and $m C_{1 v v}, m C_{2 v v}$ are the minimal value of averaged concentration $C_{1 v v}, C_{2 v v}$ in every layers, obtained in the time moment $t_{f}$. For $t_{f}=30000$ these values are equal to $10^{-5}$. For increasing $\lambda$ the concentration of water vapour in the air spaces can be increased (see Table 1).

Table 1. The values of $m C_{1 v v}, m C_{2 v v}, M C_{v}, M C$

\begin{tabular}{lllllll}
\hline$t_{f}$ & $m C_{1 v v}$ & $m C_{2 v v}$ & $M C_{v}$ & $M C$ & $\lambda$ & $\nu$ \\
\hline 1000 & 0.776 & 0.160 & 0.868 & 0.909 & 0 & 1 \\
2000 & 0.571 & 0.041 & 0.668 & 0.824 & - & - \\
5000 & 0.233 & 0.010 & 0.275 & 0.339 & - & - \\
8000 & 0.095 & 0.004 & 0.112 & 0.138 & - & - \\
10000 & 0.052 & 0.002 & 0.061 & 0.076 & - & - \\
20000 & 0.003 & .0001 & 0.003 & 0.004 & - & \\
1000 & 0.843 & 0.177 & 0.955 & 1.170 & 0.1 & 1 \\
5000 & 0.257 & 0.011 & 0.302 & 0.373 & 0.1 & 1 \\
\hline
\end{tabular}

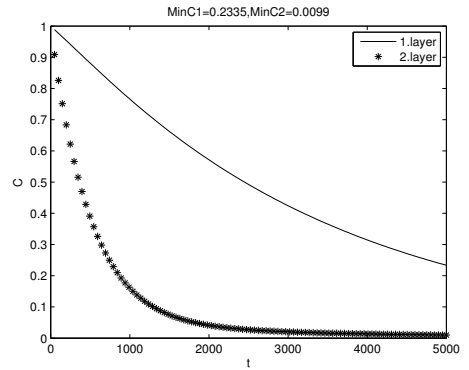

(a) values $C_{1 v v}, C_{2 v v}$ depending on $t$

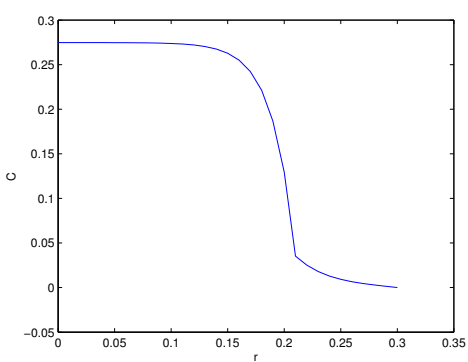

(b) values $C_{v v}$ depending on $r$ by $t=$ 5000

Figure 7. The averaged values for drying process

In following Table 2 we can see the depending $m C_{1 v v}, m C_{2 v v}, M C_{v}=M C$ on $D_{1 z}^{C}=D_{1 r}^{C}, D_{2 z}^{C}=D_{2 r}^{C}, D_{1 z}^{T}=D_{1 r}^{T}, D_{2 z}^{T}=D_{2 r}^{T}$, by $\lambda=0.1, \nu=9$, $t_{f}=10000$ and $\frac{\partial T_{i}(r, L, t)}{\partial z}=\frac{\partial C_{i}(r, L, t)}{\partial z}=0\left(M T_{v}=\max T \approx 1\right)$.

In Figs. $6 \mathrm{a}$ and $8 \mathrm{~b}$ we represent also the averaged concentration $T_{1 v v}, T_{2 v v}$ depending on time in every layer and $T_{v}=T$ depending on $r$, obtained in the time moment $t_{f}=1000$, by $D_{1 z}^{C}=D_{1 r}^{C}=10^{-5}, D_{2 z}^{C}=D_{2 r}^{C}=10^{-4}$, $D_{1 z}^{T}=D_{1 r}^{T}=10^{-4}, D_{2 z}^{T}=D_{2 r}^{T}=10^{-3}$. 


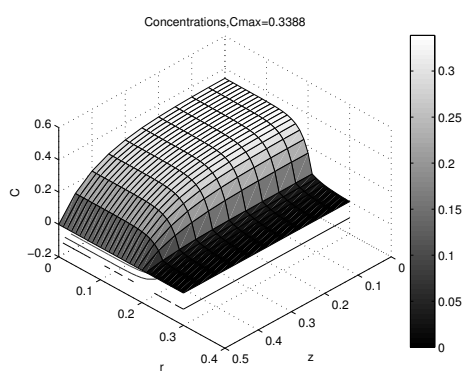

(a) $C$ depending on $(r, z)$ by $t=5000$

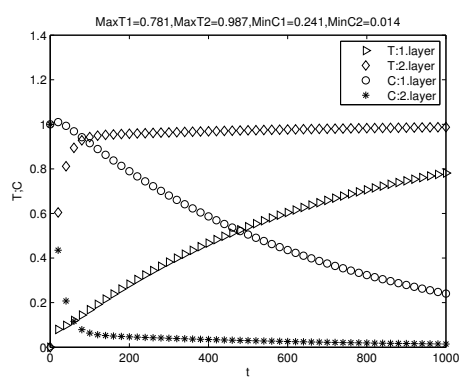

(b) the averaged values $T_{v v}, C_{v v}$ depending on $t$

Figure 8. Values for drying process

Table 2. The minimal and maximal values of temperature for $\lambda=0.1, \nu=9$

\begin{tabular}{lllllll}
\hline$D_{1 r}^{C}$ & $D_{2 r}^{C}$ & $D_{1 r}^{T}$ & $D_{2 r}^{T}$ & $m C_{1 v v}$ & $m C_{2 v v}$ & $M C_{v}$ \\
\hline $10^{-7}$ & $10^{-6}$ & $10^{-3}$ & $.510^{-3}$ & .2294 & .0135 & .4227 \\
- & - & $10^{-4}$ & $10^{-5}$ & .2321 & .0137 & .4277 \\
- & - & $10^{-5}$ & $10^{-4}$ & .2306 & .0136 & .4250 \\
$10^{-6}$ & $10^{-6}$ & $10^{-5}$ & $10^{-4}$ & .0015 & .0004 & .0020 \\
$10^{-6}$ & $10^{-7}$ & - & - & .4872 & .2180 & .5125 \\
$10^{-5}$ & $10^{-7}$ & - & - & .4668 & .2201 & .4693 \\
$10^{-7}$ & $10^{-6}$ & - & - & .1774 & .0012 & .3472 \\
$10^{-7}$ & $10^{-7}$ & - & - & .6470 & .1875 & .8820 \\
\hline
\end{tabular}

In the Table 3 are given maximal and minimal values of temperature and concentration $M T, m T, M C, m C$. We see that if parameter $\lambda$ is increased, then inside the layers the concentration of water vapour in the air spaces is also increased.

Table 3. The maximal and minimal values for $t_{f}=1000$

\begin{tabular}{llllllll}
\hline$\lambda$ & $\nu$ & $m C_{1 v v}$ & $m C_{2 v v}$ & $M C_{v}$ & $M T_{1 v v}$ & $M T_{2 v v}$ & $m T_{v}$ \\
\hline 0.1 & 9.0 & .2407 & .0142 & .4434 & .7814 & .9871 & .5972 \\
0.2 & 4.5 & .2627 & .0155 & .4841 & .8807 & .9930 & .7802 \\
\hline
\end{tabular}

\section{Conclusions}

For the transfer of moisture content and heat in porous multi-layered 3-D domain is considered the system of two PDEs for determination the concentration $C$ of water vapour in the air spaces and the temperature $T$.

The approximation of corresponding initial boundary-value problem of the system of PDEs is based on the conservative averaging method (CAM), where 
is used with new hyperbolic type splines. For these splines the best parameter for the minimal error is calculated.

For the stationary 1D boundary value problem in one and two layers the spline parameters for error corrections are caculated with minimizing method using Matlab.

The problem of the system of PDEs with constant coefficients are reduced to the initial value problem of a system of ODEs of the first order. Such a procedure allows us to obtain analytical solution with a simple engineering algorithm for mass transfer equations for different substances in layered domain. It is possible to model round and angular wood-blocks and give some new physical conclusions about the drying an also about moistening processes in this blocks.

\section{Acknowledgement}

This work was partially supported by the grant $623 / 2014$ of the Latvian Council of Science.

\section{References}

[1] M. Buike and A. Buikis. Modelling 3-D transport processes in anisotropic layered stratum by conservative averaging method. WSEAS Transactions on Heat and Mass Transfer, 1(4):430-437, 2006.

[2] M. Buike, A. Buikis and H. Kalis. Time direct and time inverse problems for wave energy and steel quenching models, solved exactly and approximately. WSEAS Transactions on Heat and Mass Transfer, 10:30-43, 2015.

[3] A. Buikis. Modelling of Filtration Processes in Layered Porous Media by the Conservative Averaging Method. Kazan, 1987. 374 p., Dr. Thesis. In Russian, 1987.

[4] A. Buikis. Definition and calculation of a generalized integral parabolic spline. Proceedings of the Latvian Academy of Sciences. Section B, 7/8(576/577):97100, 1995.

[5] A. Buikis and M. Buike. The conservative averaging method: applications, theory and new hyperbolic approximation. In North Atlantic University Union Imre J.Rudas(Ed.), Mathematical and Computation Methods in Applied Sciences,Proc. of 3-rd Int. Conf. on Applied, Numerical and Computational Mathematics(ICANCM'15), Sliema,Malta Aug. 17-19, 2015, volume 51 of Mathematics and Computers in Science and Engineering Series 51, pp. 58-67, ISSN: 22274588, ISBN:978-1-61804-328-3, 2015. WSEAS Press.

[6] A. Buikis, J. Cepitis, H. Kalis and A. Reinfelds. Non-isothermal mathematical model of wood and paper drying. In Progress in Industrial Mathematics at ECMI 2000, pp. 488-492. Springer, 2002.

[7] A. Buikis, J. Cepitis, H. Kalis and A. Reinfelds. Mathematical model of save timber drying. Proceeding of the Latvian Academy of Sciences, Sec B, $\mathbf{5 7}(3 / 4(626)): 128-132,2003$.

[8] A. Buikis, J. Cepitis and S. Kostjukova. The mathematical model of the plywood production. In Applied and Computational Mathematics. Proc. of the 
13-th WSEAS International Conference and Applied Mathematics, pp. 365-369. WSEAS Press, 2008.

[9] A. Buikis and H. Kalis. Hyperbolic type approximation for the solutions of the hyperbolic heat conduction equation in 3-D domain. In North Atlantic University Union Imre J.Rudas(Ed.), Mathematical and Computation Methods in Applied Sciences,Proc. of 3-rd Int. Conf. on Applied, Numerical and Computational Mathematics(ICANCM'15), Sliema,Malta Aug. 17-19, 2015, volume 51 of Mathematics and Computers in Science and Engineering Series 51, pp. 42-51, ISSN: 2227-4588, ISBN:978-1-61804-328-3, 2015. WSEAS Press.

[10] A. Buikis, H. Kalis and I. Kangro. Special hyperbolic type spline for mass transfer problems in multi-layer 3-D domains. In North Atlantic University Union Imre J.Rudas(Ed.), Mathematical and Computation Methods in Applied Sciences,Proc. of 3-rd Int. Conf. on Applied, Numerical and Computational Mathematics(ICANCM'15), Sliema,Malta Aug. 17-19, 2015, volume 51 of Mathematics and Computers in Science and Engineering Series 51, pp. 25-34, ISSN: 22274588, ISBN:978-1-61804-328-3, 2015. WSEAS Press.

[11] A. Buikis, H. Kalis and I. Kangro. Special splines of exponential type for the solutions of mass transfer problems in multilayer domains. Mathematical Modelling and Analysis, 21(4):450-465, 2016. https://doi.org/10.3846/13926292.2016.1182594.

[12] R. Čiegis and V. Starikovičius. The finite difference scheme for $3 \mathrm{~d}$ mathematical modelling of wood drying process. Computational methods in applied mathematics, 1(2):123-137, 2001. https://doi.org/10.2478/cmam-2001-0009.

[13] R. Čiegis and V. Starikovičius. The finite difference scheme for wood drying process. Mathematical Modelling and Analysis, 6(1):48-57, 2001.

[14] R. Čiegis and V. Starikovičius. Mathematical modelling of wood drying process. Mathematical Modelling and Analysis, 7(2):177-190, 2002.

[15] J. Crank. The mathematics of diffusion. Oxford, at the Clarendon Press, 1956.

[16] H. Kalis and I. Kangro. Calculation of heat and moisture sistribution in the porous media layer. Mathematical Modelling and Analysis, 12(1):91-100, 2007. https://doi.org/10.3846/1392-6292.2007.12.91-100.

[17] M. Kaviany. Principles of heat transfer in porous media. Springer-Verlag, Berlin, 1991. https://doi.org/10.1007/978-1-4684-0412-8.

[18] S. Kostjukova and A. Buikis. Mathematical model for diffusion-sorption processes in layered strata with interlayers. Environmental Science, Ecosystems and Development, WSEAS Press, pp. 7-12, 2007.

[19] R.Čiegis and O. Iliev. On numerical simulation of liquid polymer moulding. Mathematical Modelling and Analysis, 8(3):101-202, 2003.

[20] J. Salin. Modelling of wood drying. Drying Technology, 9(3):775-793, 1991. https://doi.org/10.1080/07373939108916709. 